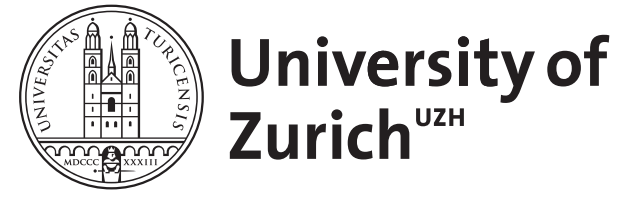

\title{
Epigenetics in rheumatoid arthritis
}

\author{
Klein, Kerstin ; Gay, Steffen
}

\begin{abstract}
PURPOSE OF REVIEW To give an overview of recently published articles addressing the role of epigenetic modifications in rheumatoid arthritis (RA). Here we focused on DNA methylation and posttranslational histone modifications. RECENT FINDINGS Recent studies attempted to link epigenetic modifications with genetic or environmental risk factors for RA. There is evidence that histone deacetylases confer effects of environmental triggers such as smoking, diet or therapy on expression levels of target genes. Additionally, disturbed methylation patterns and cell-type specific histone methylation marks were identified as potential mediators of genetic risk in RA. Altered methylome signatures were found in several cell types in RA, first of all RA synovial fibroblasts, and contribute to the intrinsic fibroblast activation. The reversal of DNA hypomethylation by inhibiting the polyamine recycling pathway was suggested as new epigenetic therapy in RA. Moreover, targeting epigenetic reader proteins, such as bromodomain proteins, emerged as a new field in drug development and the first studies underscored the potential of these drugs not only in malignant and inflammatory conditions but also in autoimmune diseases. SUMMARY Epigenetic factors represent a promising area to link genetics, regulation of gene expression and environmental risk factors.
\end{abstract}

DOI: https://doi.org/10.1097/BOR.0000000000000128

Posted at the Zurich Open Repository and Archive, University of Zurich ZORA URL: https://doi.org/10.5167/uzh-109207

Journal Article

Published Version

Originally published at:

Klein, Kerstin; Gay, Steffen (2015). Epigenetics in rheumatoid arthritis. Current Opinion in Rheumatology, 27(1):76-82.

DOI: https://doi.org/10.1097/BOR.0000000000000128 


\title{
Epigenetics in rheumatoid arthritis
}

\author{
Kerstin Klein and Steffen Gay
}

\begin{abstract}
Purpose of review
To give an overview of recently published articles addressing the role of epigenetic modifications in rheumatoid arthritis (RA). Here we focused on DNA methylation and posttranslational histone modifications.

\section{Recent findings}

Recent studies attempted to link epigenetic modifications with genetic or environmental risk factors for RA. There is evidence that histone deacetylases confer effects of environmental triggers such as smoking, diet or therapy on expression levels of target genes. Additionally, disturbed methylation patterns and cell-type specific histone methylation marks were identified as potential mediators of genetic risk in RA. Altered methylome signatures were found in several cell types in RA, first of all RA synovial fibroblasts, and contribute to the intrinsic fibroblast activation. The reversal of DNA hypomethylation by inhibiting the polyamine recycling pathway was suggested as new epigenetic therapy in RA. Moreover, targeting epigenetic reader proteins, such as bromodomain proteins, emerged as a new field in drug development and the first studies underscored the potential of these drugs not only in malignant and inflammatory conditions but also in autoimmune diseases.
\end{abstract}

\section{Summary}

Epigenetic factors represent a promising area to link genetics, regulation of gene expression and environmental risk factors.

\section{Keywords}

bromodomain protein, DNA methylation, histone, rheumatoid arthritis

\section{INTRODUCTION}

Rheumatoid arthritis (RA) is an autoimmune disease, characterized by chronic inflammation of the joints with severe pain and swelling, joint damage and disability, which leads ultimately to joint destruction and loss of function. RA can be divided into two major subsets based on the presence or absence of antibodies to citrullinated peptide antigens (ACPA) [1]. The best know genetic risk factor for $\mathrm{ACPA}^{+} \mathrm{RA}$ is the presence of certain human leukocyte antigen (HLA) class II alleles. Several genome-wide association studies (GWAS) have identified genetic variants across different populations that confer risk to RA. However, these variants can explain only less than $20 \%$ of susceptibility in $\mathrm{ACPA}^{+} \mathrm{RA}$ and even less in $\mathrm{ACPA}^{-} \mathrm{RA}$. The contribution of environmental triggers including smoking, the best known environmental risk factor for RA, is essential to RA pathogenesis $[2,3]$. The understanding of the interplay between immunity, life style as well as genetic and environmental factors is poor and is key to explaining the cause of RA. Epigenetic factors represent a promising area to link genetics and gene expression with disease risk [3].

\section{EPIGENETIC FACTORS AS REGULATORS OF GENE EXPRESSION}

The term 'epigenetics' was originally associated with heritable changes in gene activity that occur without alterations of the genetic code. Nowadays, the term is more often used to describe chromatinrelated regulatory mechanisms that do not involve changes in the nucleotide sequence, regardless of whether such imprinting is strictly heritable [4]. Posttranslational histone modifications such as acetylation, methylation or phosphorylation marks among others are added to histone side chains or to cytosine residues in the DNA (DNA methylation) by epigenetic 'writer' proteins (Fig. 1). These epigenetic marks are recognized by 'reader' proteins that serve

\footnotetext{
Center of Experimental Rheumatology, University Hospital Zurich, Zurich, Switzerland

Correspondence to Prof Steffen Gay, Center of Experimental Rheumatology, University Hospital Zurich, Gloriastrasse 25, CH-8091 Zurich, Switzerland. Tel: +41 44255 5737; fax: +41 44255 4415; e-mail: steffen.gay@usz.ch
}

Curr Opin Rheumatol 2015, 27:76-82

DOI:10.1097/BOR.0000000000000128 


\section{KEY POINTS}

- Epigenetic modifications can serve as intermediaries of genetic risk and environmental factors in RA.

- Inhibition of the polyamine recycling pathway reverses the hypomethylation in rheumatoid arthritis synovial fibroblasts (RASF) and reduces the invasiveness of RASF in vivo.

- Targeting epigenetic reader proteins such as bromodomain and extraterminal proteins might become new therapies in autoimmune diseases.

as platforms and docking sites for effector proteins mediating fundamental processes such as transcription, DNA replication and recombination, DNA damage response and chromatin remodeling. Epigenetic 'eraser' proteins are capable of removing epigenetic marks. Understanding the intricate spatial and temporal interplay between different epigenetic modifications is one of the most challenging aspects in chromatin biology and at the beginning of exploration [4].

In addition to posttranslational histone modifications and DNA methylation, noncoding RNAs are additional factors that influence gene expression

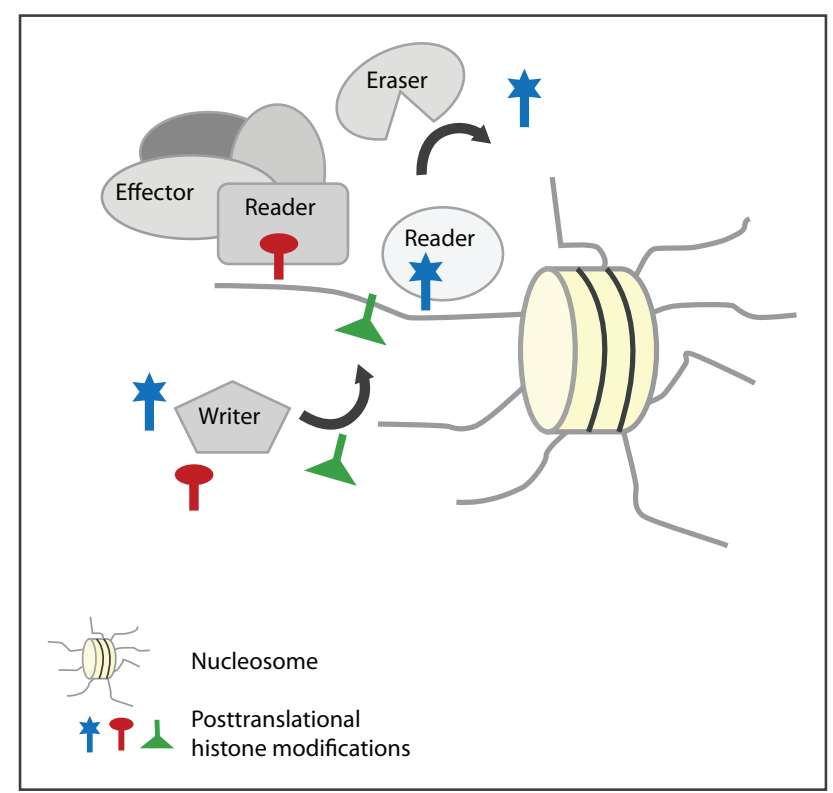

FIGURE 1. Epigenetic writer, reader and eraser proteins. Posttranslational histone modifications such as acetylation, methylation or phosphorylation marks are added to histone side chains by writer proteins and are recognized and get bound by reader proteins. Epigenetic reader proteins serve as a platform and docking site for effector proteins.

Posttranslational histone modifications are removed by eraser proteins. levels. Apart from microRNAs [5], which are small noncoding RNAs acting as destabilizers and repressors of translation, the functional role of other noncoding RNAs in RA has not been investigated yet. Long noncoding RNAs with a length of at least 200 nucleotides regulate gene expression levels by affecting the stability of mRNA, altering the translation efficiency, and functioning as precursors for mRNAs. Furthermore, long noncoding RNAs are capable of controlling the epigenetic state of particular genes by guiding chromatinmodifying complexes and other nuclear proteins to specific genomic loci to exert their effects [6]. Song et al. [7] have recently shown an altered pattern of long noncoding RNAs in peripheral blood mononuclear cells (PBMC) of RA patients compared with healthy controls but their impact on disease pathogenesis is at the beginning of investigation.

\section{DNA METHYLATION}

Methylation of DNA is carried out by the activity of DNA methyl transferases (DNMT), leading to the formation of 5-methylcytosine (5-mC). Most research so far has focused on the role of 5-mC in $\mathrm{CpG}$ rich regions, called $\mathrm{CpG}$ islands. They are enriched in promoters in the vicinity of transcriptional start sites and their methylation blocks the initiation of transcription and is associated with long-term gene silencing including X-chromosome inactivation [8].

An altered DNA methylome signature in synovial fibroblasts derived from RA and osteoarthritis patients, in PBMC from RA patients and healthy controls, as well as reduced amounts of $5-\mathrm{mC}$ in synovial tissues of RA patients were described by different research groups $\left[9,10,11^{\prime}\right]$. Hypomethylated genes clustered in key pathways related to cell migration, including focal adhesion, cell adhesion, transendothelial migration and extracellular matrix interactions [11"]. Karouzakis et al. [12"] showed that increased levels of the polyamine-modulated factor 1-binding protein 1 (PMFBP1) and the spermidine/ spermine N1-acetyltransferase (SSAT1) in rheumatoid arthritis synovial fibroblasts (RASF) enhanced the catabolism and recycling of polyamines. The authors suggested that the high consumption of $S$-adenosyl methionine (SAM), the methyl donor during DNA methylation via this pathway as an important factor contributing to the global DNA hypomethylation in RASF [12"]. In a follow-up study, Neidhart et al. [13"'] recently suggested a new therapeutic concept for inhibiting the ongoing joint destruction in RA based on the inhibition of the polyamine recycling pathway by using diminazene 
aceturate, an inhibitor of the SSAT-1, alone, or in combination with SAM/L-methionine. The authors showed that SSAT-1 inhibition reduced the adhesion abilities and expression levels of matrix metalloproteinase (MMP) 1 in RASF as well as the invasiveness of RASF that were coimplanted with cartilage into the severe combined immunodeficiency mouse model [13"']. This would be the first therapy targeting RASF.

Besides global changes in DNA methylation, promoters of specific genes were shown to be hypomethylated or hypermethylated in RA in different cell types $\left[14,15,16^{\mathbf{*}}, 17\right]$. The methylation of a single $\mathrm{CpG}$ site in the promoter of cytotoxic T-lymphocyte-associated protein 4 (CTLA-4) in regulatory $\mathrm{T}$ cells (Treg) of RA patients was shown to result in impaired binding of the transcription factor nuclear factor of activated T cells 2 (NFAT2) leading to decreased expression levels of CTLA-4 in RA patients compared with healthy controls. As a consequence, Treg were unable to activate the tryptophan-degrading enzyme indoleamine 2,3-dioxygenase and therefore failed to activate the immune modulatory kynurenine pathway [16"]. These results implicate that small epigenetic changes can impact the function of a whole cell type in RA, in this case Treg.

\section{HISTONE ACETYLATION}

Histone acetylation and deacetylation are executed by the activity of two opposing enzyme families: histone acetylases (HAT) and histone deacetylases (HDAC). HDAC exert the epigenetic control of transcriptional activity by removing negatively charged acetyl groups from lysine residues of histones leading to the condensation of chromatin and reducing the accessibility for transcription factors. The human genome contains 18 genes encoding for HDAC that are classified into four groups based on structural and functional similarities. Whereas class I HDAC (HDAC 1, 2, 3, 8) are widely expressed and present in nuclei, class II HDAC (HDAC 4-7, 9) exhibit a more limited expression pattern and are able to shuttle between the nucleus and the cytoplasm. Sirtuins (SIRT 1-7) are members of class III HDAC and require $\mathrm{NAD}^{+}$for their enzymatic activity. HDAC11 is the sole member of class IV HDAC [18]. In addition to dietary factors that are known to affect HDAC activity [19], treatment of RA patients with antitumor necrosis factor (TNF) $\alpha$ therapy was shown to increase the HAT/HDAC ratio in nuclear extracts of PBMC, whereas rituximab increased nuclear activity of both HAT and HDAC [20]. Recently, direct effects of smoking on changes in gene expression levels in joints were described $\left[21^{-*}, 22^{*}\right]$ and SIRT6 was identified as one of the mediators of smoke-induced changes in RASF. Engler et al. [22"] showed that SIRT6 levels were increased in synovial tissues of smokers and SIRT6 levels correlated with the disease duration in smokers but not in nonsmokers. They showed that the up-regulation of SIRT6 in RASF under exposure to cigarette-smoke extract or $\mathrm{TNF} \alpha$ functions as counterregulatory mechanism attenuating the production of MMP1 [22"]. These findings were in line with a previous report revealing an anti-inflammatory and antidestructive role of SIRT6 in RASF in vitro and in the collagen-induced arthritis (CIA) mouse model in vivo [23"']. On the contrary, SIRT1, expression levels of which are increased in synovial tissues and synovial cells of RA patients, was shown to have proinflammatory properties and contributes to the apoptosis-resistant phenotype seen in RASF [24]. These reciprocal functions of SIRT1 and SIRT6 in RASF underscore the importance of performing functional studies on individual members of HDAC families.

Different HDAC inhibitors (Table 1), which mostly target several members of HDAC, were described to have beneficial effects in vitro and in vivo in RA $[17,25-31]$. However, many of the observed effects of HDAC inhibitors were shown to be chromatin-independent and nonepigenetic (reviewed in [17]). Further studies [29-31] (Table 1) have been published within the last 2 years using new and even more potent HDAC inhibitors. Ahmed et al. [29] showed that Largazole (LAR), a marine-derived class I HDAC inhibitor, suppressed the TNF $\alpha$-induced expression of intracellular adhesion molecule-1 (ICAM-1) and vascular adhesion molecule-1 (VCAM-1) in RASF, whereas the TNF $\alpha$-induced MMP2 activity was reduced. Furthermore, the authors showed that LAR modulated expression levels of HDAC1, HDAC5 and HDAC6 and indicated a role of HDAC6 in the LAR-induced changes of ICAM-1 and VCAM-1 expression levels. These results again point to the need for understanding the exact role of different HDAC enzymes in the RA pathogenesis in order to develop highly effective HDAC inhibitors for the treatment of RA [29].

\section{EPIGENETIC MECHANISMS AS AN INTERMEDIARY OF GENETIC RISK}

Recent research efforts tried to integrate epigenetic mechanisms into genetic susceptibility loci as causes of the disease [32"',33"']. Liu et al. [32"'] performed a genome-scale methylation and single nucleotide polymorphism (SNP) analysis in leukocytes derived from $\mathrm{ACPA}^{+}$patients. The authors identified nine 


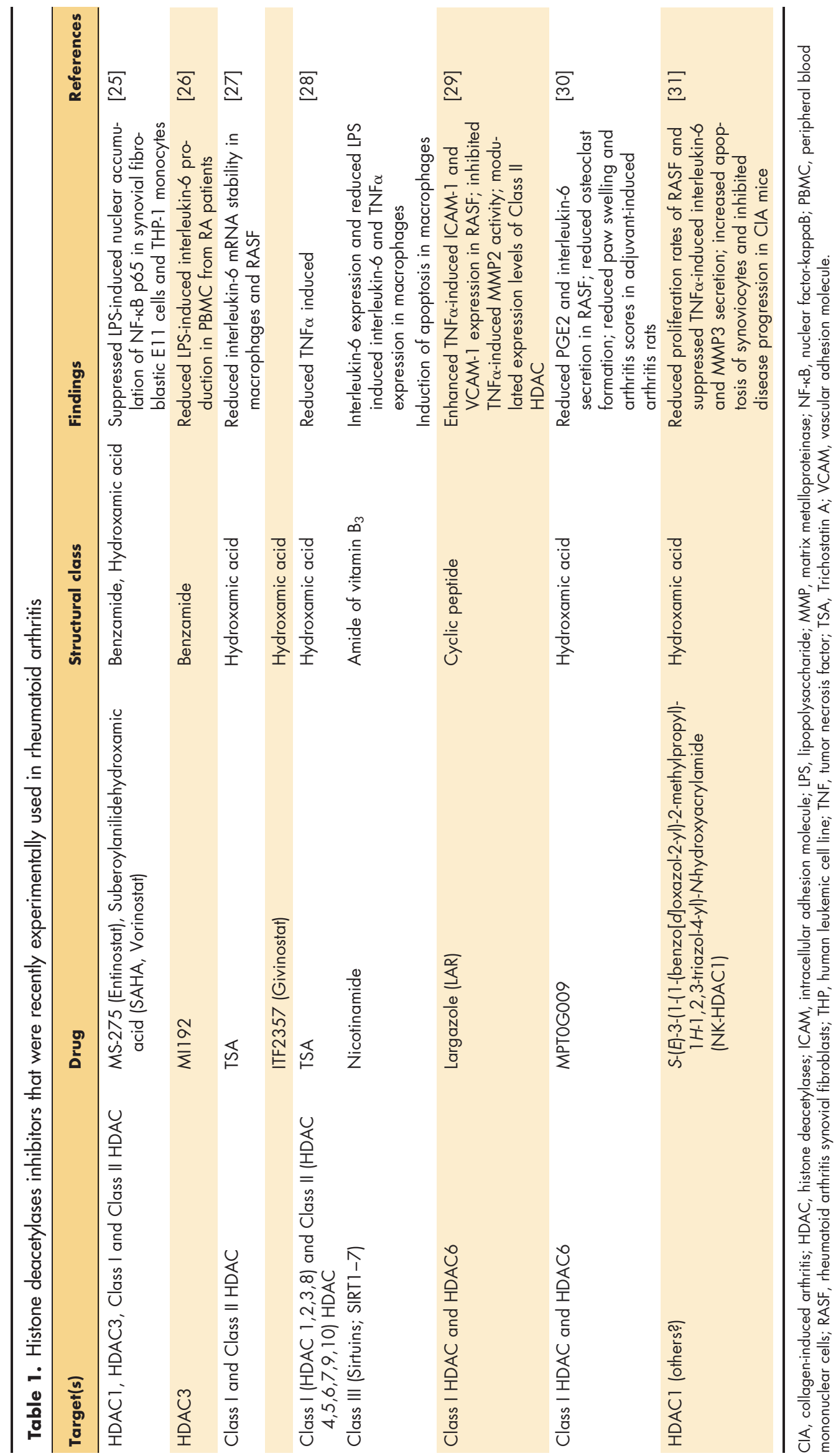


clusters of differentially methylated signatures in the MHC region and one outside on the same chromosome that likely mediate the genetic risk for RA [32"-]. Furthermore, in a three-stage transethnic meta-analysis of GWAS data, an enrichment of non-MHC risk loci was assessed in epigenetic chromatin marks [33"']. Histone 3 lysine 4 trimethylation (H3K4me3), a mark that highlights active promoters and enhancers, was previously shown to be the histone mark that is most phenotypically celltype specific [34]. RA risk loci in biological candidate genes overlapped with H3K4me3 peaks in immunerelated cells, especially in Treg [33"'].

\section{OTHER POSTTRANSLATIONAL HISTONE MODIFICATIONS}

Only a few studies analyzed the role of other posttranslational histone modifications than acetylation in RA (reviewed in [17]). Recently, the first study on histone phosphorylation in arthritis was published. The authors identified an overexpression of Aurora kinases A (AURKA) and B (AURKB) in mononuclear cells derived from proteoglycan-induced arthritis (PGIA) mice and PBMC derived from treatment naive RA patients compared with healthy controls by screening 84 known chromatin-modifying enzymes. Increased AURKA and AURKB levels in B cells derived from PGIA mice correlated with elevated levels of phosphorylated histone 3 . Treatment with a pan-aurora kinase inhibitor promoted B-cell apoptosis and attenuated inflammatory reactions in arthritic mice [35"].

\section{TARGETING EPIGENETIC READER PROTEINS}

In recent years, much effort was put into the development of small molecule inhibitors of the bromodomain and extraterminal (BET) family (BRD2, BRD3, BRD4, BRDT) of bromodomain proteins. BET proteins are readers of the $\varepsilon$ - $N$-acetylation of lysine residues (Kac) on histone tails, a modification that is associated with an open chromatin architecture and transcriptional activation [36]. First clinical trials using the BET inhibitors I-BET762 for the treatment of nuclear protein in testis (NUT) midline carcinomas, OTX015 for the treatment of hematological malignancies and CPI-0610 for the treatment of lymphomas have started recently (reviewed in [37]), underscoring the potential of this class of inhibitors. However, BET inhibition as a point of therapeutic intervention also became of interest in autoimmune conditions because of the strong antiinflammatory properties of BET inhibitors [38,39]. A first hint for an association of BET proteins with RA came from a study by Mahdi et al. [40] in which specific interactions between genotype, smoking and autoimmunity to citrullinated $\alpha$-enolase were analyzed. Three SNPs in the BRD2 locus were found to be associated with a subset of RA patients positive for citrullinated $\alpha$-enolase peptide 1 and cyclic citrullinated peptides, independently of the HLADRB1 shared epitope alleles [40]. In addition, a SNP in the locus of another epigenetic reader protein, namely BRD1 (BRPF2) was shown to be protective in joint damage progression in stage I of a GWAS in $\mathrm{ACPA}^{+}$RA patients [41]. Recently, therapeutic dosing of the BET inhibitor JQ1 was proven to be

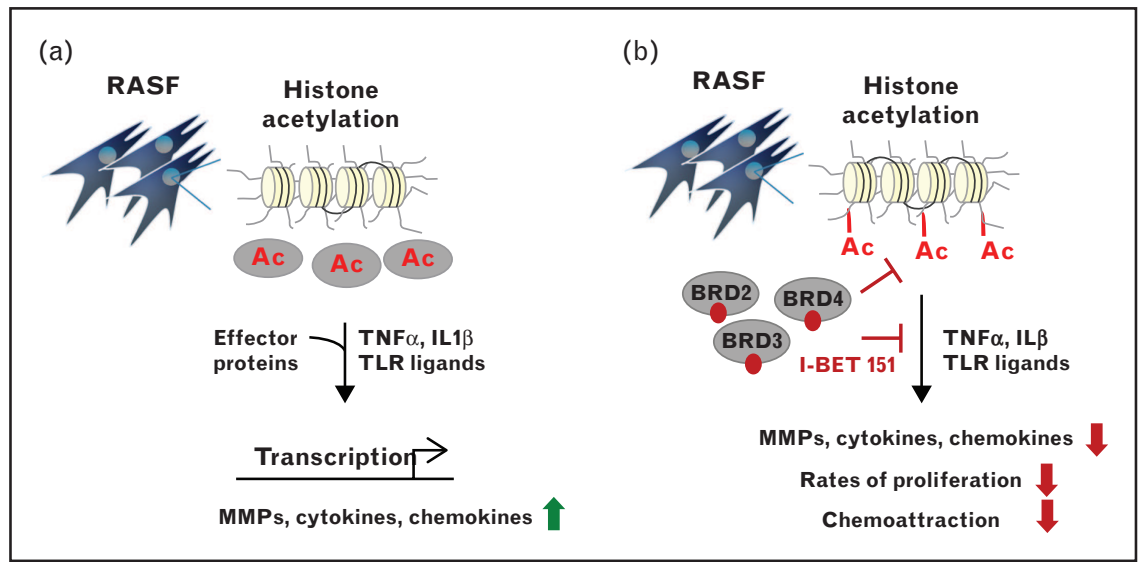

FIGURE 2. Targeting epigenetic reader proteins with I-BET151. (a) Acetylated side chains of histones are recognized by the epigenetic reader proteins BRD2, BRD3 and BRD4, leading to the recruitment of effector proteins and the activation of transcription. (b) I-BET151 is a histone mimic that prevents BRD2, BRD3, and BRD4 from binding to acetylated histone side chains, suppresses the transcription of matrix degrading enzymes, inflammatory cytokines and chemokines after stimulation with TNF $\alpha$, interleukin-1 $\beta$ or Toll-like receptor (TLR) ligands and reduces proliferation rates and the chemoattractive potential of RASF. BET, bromodomain and extraterminal; RASF, rheumatoid arthritis synovial fibroblasts; TNF, tumor necrosis factor. 
efficacious in two mouse models of autoimmunity, the models for CIA and for experimental autoimmune encephalomyelitis (EAE); BET inhibition suppressed the differentiation and activation of Th17 cells [42"']. Furthermore, we have shown that the inhibitor I-BET151 suppressed the expression of a broad range of inflammatory mediators and matrix degrading enzymes in RASF in the presence of cytokines or Toll-like receptor (TLR) ligands (Fig. 2). Furthermore, I-BET151 reduced proliferation rates of RASF and chemotactic properties towards PBMC (Klein et al., unpublished). However, the individual roles of BET proteins in mediating effects of different BET inhibitors in inflammatory and autoimmune conditions has hardly been investigated yet.

Besides targeting bromodomain proteins [37], readers of other epigenetic marks including selected members of methyl-lysine binding site readers were predicted to have good drugability and first inhibitors are in development [43].

\section{CONCLUSION}

The comprehension of the role of epigenetic factors contributing to the pathogenesis of RA has increased in recent years. A future challenge is to reveal the interplay between different epigenetic modifications and to implement more functional studies in order to understand underlying mechanisms of epigenetic factors in different cell types contributing to RA pathogenesis.

\section{Acknowledgements}

None.

\section{Financial support and sponsorship}

This work was supported by a grant of IAR, and by the IMI funded project BeTheCure (115142-2).

\section{Conflicts of interest}

None.

\section{REFERENCES AND RECOMMENDED \\ READINC}

Papers of particular interest, published within the annual period of review, have been highlighted as:

- of special interest

- of outstanding interest

1. Padyukov L, Seielstad M, Ong RT, et al. A genome-wide association study suggests contrasting associations in ACPA-positive versus ACPA-negative rheumatoid arthritis. Ann Rheum Dis 2011; 70:259-265.

2. Klareskog L, Gregersen PK, Huizinga TW. Prevention of autoimmune rheumatic disease: state of the art and future perspectives. Ann Rheum Dis 2010; 69:2062-2066.

3. Viatte $S$, Plant $D$, Raychaudhuri $S$. Genetics and epigenetics of rheumatoid arthritis. Nat Rev Rheumatol 2013; 9:141-153.

4. Musselman CA, Lalonde ME, Cote J, et al. Perceiving the epigenetic landscape through histone readers. Nat Struct Mol Biol 2012; 19:1218-1227.
5. Filkova M, Jungel $A$, Gay RE, et al. MicroRNAs in rheumatoid arthritis: potential role in diagnosis and therapy. BioDrugs 2012; 26:131-141.

6. Zhang $\mathrm{K}$, Shi ZM, Chang YN, et al. The ways of action of long noncoding RNAs in cytoplasm and nucleus. Gene 2014; 547:1-9.

7. Song J, Kim D, Han J, et al. PBMC and exosome-derived Hotair is a critical regulator and potent marker for rheumatoid arthritis. Clin Exp Med 2014; Epub ahead of print.

8. Jones PA. Functions of DNA methylation: islands, start sites, gene bodies and beyond. Nat Rev Genet 2012; 13:484-492.

9. Karouzakis E, Gay RE, Michel BA, et al. DNA hypomethylation in rheumatoid arthritis synovial fibroblasts. Arthritis Rheum 2009; 60:3613-3622.

10. Liu CC, Fang TJ, Ou TT, et al. Global DNA methylation, DNMT1, and MBD2 in patients with rheumatoid arthritis. Immunol Lett 2011; 135:96-99.

11. Nakano $\mathrm{K}$, Whitaker JW, Boyle DL, et al. DNA methylome signature in

- rheumatoid arthritis. Ann Rheum Dis 2013; 72:110-117.

The authors provide a genome-wide evaluation of DNA methylation patterns in

RASF that distinguish them from osteoarthritis and normal synovial fibroblasts.

12. Karouzakis E, Gay RE, Gay S, et al. Increased recycling of polyamines is

- associated with global DNA hypomethylation in rheumatoid arthritis synovial fibroblasts. Arthritis Rheum 2012; 64:1809-1817.

The authors provide a concept how an increased polyamine metabolism in rheumatoid arthritis synovial fibroblasts causes global DNA hypomethylation and contributes to the intrinsic activation of these cells.

13. Neidhart M, Karouzakis $E$, Jungel $A$, et al. Inhibition of spermidine/spermine

- n1-acetyltransferase activity: a new therapeutic concept in rheumatoid arthritis. Arthritis Rheumatol 2014; 66:1723-1733.

The authors suggest a new therapeutic concept for RA based on the inhibition of polyamine recycling which reverses the global DNA hypomethylation in RASF.

14. Park SH, Kim SK, Choe JY, et al. Hypermethylation of EBF3 and IRX1 genes in synovial fibroblasts of patients with rheumatoid arthritis. Mol Cells 2013; 35:298-304.

15. Zhou Q, Long L, Shi G, et al. Research of the methylation status of miR-124a gene promoter among rheumatoid arthritis patients. Clin Dev Immunol 2013; 2013:524204.

16. Cribbs AP, Kennedy $A$, Penn $H$, et al. Regulatory $T$ cell function in rheumatoid

- arthritis is compromised by CTLA-4 promoter methylation resulting in a failure to activate the IDO pathway. Arthritis Rheumatol 2014; 66:2344-2354.

The authors demonstrate how the methylation of a single site in the promoter of the CTLA-4 gene leads to defective function of Tregs in RA.

17. Klein $\mathrm{K}$, Gay S. Epigenetic modifications in rheumatoid arthritis, a review. Curr Opin Pharmacol 2013; 13:420-425.

18. McGee-Lawrence ME, Westendorf JJ. Histone deacetylases in skeletal development and bone mass maintenance. Gene 2011; 474:1-11.

19. Rajendran $P$, Williams DE, Ho $E$, et al. Metabolism as a key to histone deacetylase inhibition. Crit Rev Biochem Mol Biol 2011; 46:181-199.

20. Toussirot $E$, Wendling $D$, Herbein $G$, et al. Biological treatments given in patients with rheumatoid arthritis or ankylosing spondylitis modify HAT/HDAC (histone acetyltransferase/histone deacetylase) balance. Joint Bone Spine 2014; Epub ahead of print

21. Ospelt $C$, Camici GG, Engler $A$, et al. Smoking induces transcription of the heat

-1. shock protein system in the joints. Ann Rheum Dis 2014; 73:1423-1426.

This is the first study showing direct effects of smoking on gene expression levels in joints.

22. Engler A, Niederer F, Klein K, et al. SIRT6 regulates the cigarette smoke-

- induced signalling in rheumatoid arthritis synovial fibroblasts. J Mol Med (Berl) $2014 ; 92: 757-767$.

The authors show that Sirtuin 6 is a mediator of cigarette-smoke-induced changes in gene expression in RASF.

23. Lee HS, Ka SO, Lee SM, et al. Overexpression of sirtuin 6 suppresses

I. inflammatory responses and bone destruction in mice with collagen-induced arthritis. Arthritis Rheum 2013; 65:1776-1785.

The authors demonstrate anti-inflammatory and antidestructive effects of Sirtuin 6 in RASF in vitro and in CIA in vivo.

24. Niederer F, Ospelt C, Brentano F, et al. SIRT1 overexpression in the rheumatoid arthritis synovium contributes to proinflammatory cytokine production and apoptosis resistance. Ann Rheum Dis 2011; 70:1866-1873.

25. Choo QY, Ho PC, Tanaka Y, et al. Histone deacetylase inhibitors MS- 275 and SAHA induced growth arrest and suppressed lipopolysaccharide-stimulated NF-kappaB p65 nuclear accumulation in human rheumatoid arthritis synovial fibroblastic E11 cells. Rheumatology (Oxford) 2010; 49:1447-1460.

26. Gillespie J, Savic S, Wong $C$, et al. Histone deacetylases are dysregulated in rheumatoid arthritis and a novel histone deacetylase 3-selective inhibitor reduces interleukin- 6 production by peripheral blood mononuclear cells from rheumatoid arthritis patients. Arthritis Rheum 2012; 64:418-422.

27. Grabiec AM, Korchynskyi O, Tak PP, et al. Histone deacetylase inhibitors suppress rheumatoid arthritis fibroblast-like synoviocyte and macrophage IL-6 production by accelerating mRNA decay. Ann Rheum Dis 2012; 71:424-431.

28. Grabiec AM, Krausz $S$, de Jager $W$, et al. Histone deacetylase inhibitors suppress inflammatory activation of rheumatoid arthritis patient synovial macrophages and tissue. J Immunol 2010; 184:2718-2728.

29. Ahmed S, Riegsecker $S$, Beamer $M$, et al. Largazole, a class I histone deacetylase inhibitor, enhances TNF-alpha-induced ICAM-1 and VCAM-1 expression in rheumatoid arthritis synovial fibroblasts. Toxicol Appl Pharmacol 2013; 270:87-96. 
30. Hsieh IN, Liou JP, Lee HY, et al. Preclinical antiarthritic study and pharmacokinetic properties of a potent histone deacetylase inhibitor MPT0G009. Cell Death Dis 2014; 5:e1166.

31. Li M, Liu X, Sun X, et al. Therapeutic effects of NK-HDAC-1, a novel histone deacetylase inhibitor, on collagen-induced arthritis through the induction of apoptosis of fibroblast-like synoviocytes. Inflammation 2013; 36:888-896.

32. Liu Y, Aryee MJ, Padyukov L, et al. Epigenome-wide association data implicate

-1. DNA methylation as an intermediary of genetic risk in rheumatoid arthritis. Nat Biotechnol 2013; 31:142-147.

The authors identified DNA methylation as a potential mediator of genetic risk in RA.

33. Okada Y, Wu D, Trynka G, et al. Genetics of rheumatoid arthritis contributes

-1. to biology and drug discovery. Nature 2014;506:376-381.

The authors conducted a three-stage trans-ethnic meta analysis on GWAS data

from Europa and Asia and showed that risk loci for RA in candidate genes overlapped with methylation peaks in Tregs.

34. Trynka G, Sandor C, Han B, et al. Chromatin marks identify critical cell types for fine mapping complex trait variants. Nat Genet 2013; 45:124-130.

35. Glant TT, Besenyei $T$, Kadar A, et al. Differentially expressed epigenome

- modifiers, including aurora kinases $A$ and $B$, in immune cells in rheumatoid arthritis in humans and mouse models. Arthritis Rheum 2013; 65:17251735.

This is the first study investigating the role of histone phosphorylation in RA.
36. Filippakopoulos $P$, Qi J, Picaud S, et al. Selective inhibition of BET bromodomains. Nature 2010; 468:1067-1073.

37. Gallenkamp D, Gelato KA, Haendler B, et al. Bromodomains and their pharmacological inhibitors. ChemMedChem 2014; 9:438-464.

38. Nicodeme E, Jeffrey KL, Schaefer U, et al. Suppression of inflammation by a synthetic histone mimic. Nature 2010; 468:1119-1123.

39. Belkina AC, Nikolajczyk BS, Denis GV. BET protein function is required for inflammation: Brd2 genetic disruption and BET inhibitor JQ1 impair mouse macrophage inflammatory responses. J Immunol 2013; 190:3670-3678.

40. Mahdi $\mathrm{H}$, Fisher BA, Kallberg $\mathrm{H}$, et al. Specific interaction between genotype, smoking and autoimmunity to citrullinated alpha-enolase in the etiology of rheumatoid arthritis. Nat Genet 2009; 41:1319-1324.

41. Knevel R, Klein $K$, Somers $K$, et al. Identification of a genetic variant for joint damage progression in autoantibody-positive rheumatoid arthritis. Ann Rheum Dis 2013; 73:2038-2046.

42. Mele DA, Salmeron $A$, Ghosh $S$, et al. BET bromodomain inhibition supm presses TH17-mediated pathology. J Exp Med 2013; 210:2181-2190.

This is the first study showing beneficial effects of BET bromodomain inhibition in mouse models for autoimmune diseases and reveals a critical role for BET proteins in TH17-cell polarization and function.

43. Santiago C, Nguyen K, Schapira M. Druggability of methyl-lysine binding sites. J Comput Aided Mol Des 2011; 25:1171-1178. 